\title{
Giuristi ed economisti nella massoneria italiana fra le due guerre
}

\author{
Fulvio Conti*
}

\section{Questioni metodologiche e interpretative}

Il rapporto che intercorse fra massoneria e università fra Otto e Novecento, nella stagione in cui il sodalizio liberomuratorio ebbe il maggior seguito di affiliati ed esercitò larga influenza nella sfera pubblica, è rimasto fino a poco tempo fa un territorio quasi del tutto inesplorato ${ }^{1}$. Maggiore interesse ha suscitato fra gli storici l'attenzione, invero assai rilevante, che il Grande Oriente d'Italia (Goi) dedicò alla questione dell' istruzione e delle politiche scolastiche durante tutta l'età liberale. Il Goi, risorto nell'autunno del 1859 dopo la lunga eclissi risorgimentale, capì infatti che per realizzare il suo ambizioso programma di modernizzazione laica e democratica del Paese era indispensabile partire dalla scuola: innalzare l'età dell'obbligo scolastico, far sì che la frequenza diventasse realmente gratuita, abolire l'insegnamento della religione, garantire

\footnotetext{
Università degli studi di Firenze, Dipartimento di scienze politiche e sociali.

Ho cercato di colmare almeno parzialmente la lacuna con il mio Massoneria e università. Presenze liberomuratorie negli atenei italiani dall'Unità al fascismo, «Annali di storia delle università italiane», 22 (2), 2018, pp. 147-177. Il presente contributo, pur dedicando specifica attenzione alle figure del mondo giuridico ed economico fra le due guerre mondiali, riprende in larghi tratti quanto anticipato in quell'articolo.
} 
che dopo il ciclo delle scuole elementari e medie vi fosse un'offerta adeguata di studi superiori ${ }^{2}$.

Ebbene, sembra di poter escludere che la massoneria abbia riservato all' istituzione universitaria in quanto tale (al suo disciplinamento, alla sua articolazione territoriale, al trattamento del corpo docente ecc.) un'attenzione paragonabile a quella che dedicò alla scuola elementare e media. Non si può pensare, d'altro canto, che la relazione si ponesse soltanto in termini di opportunità di carriera, che la scelta di aderire alla massoneria fosse vista semplicemente come un mezzo per ottenere ruoli e promozioni. Situazioni di questo genere probabilmente vi furono, ma non è possibile ricondurre a questa sola variabile un rapporto che fu ben altrimenti complesso e che trasse origine principalmente da scelte di natura politica e culturale.

Questo contributo, muovendo da considerazioni di carattere più generale riguardanti l'intera università e il suo corpo docente, intende offrire anzitutto qualche prima indicazione sulla presenza di giuristi ed economisti nei ranghi del Grande Oriente d'Italia nel periodo fra la Prima e la Seconda guerra mondiale. Quanti furono gli accademici attivi in questi due settori disciplinari che subirono il fascino dell'iniziazione liberomuratoria? Si trattò di personaggi minori del mondo accademico oppure di figure di primo piano sia sul versante scientifico sia su quello gestionale (rettori, presidi, direttori di istituti e strutture varie)? E quanti di loro ricoprirono ruoli politici e istituzionali di rilievo nazionale? Nelle pagine che seguono, lungi dalla pretesa di fornire dati esaustivi, si cercherà, attraverso un'analisi di tipo prosopografico, di delineare un primo quadro d'insieme dei consensi che la massoneria raccolse nello specifico contesto della cultura giuridica ed economica.

Il tema riveste un certo interesse anche perché rimanda a quello più generale della cultura massonica. Com'è noto, Benedetto Croce in un'intervista rilasciata nel novembre 1910, poi raccolta in un volume di scritti più volte ristampato, bollò quella massonica come «cultura ottima per commercianti, piccoli professionisti, maestri elementari, avvocati, mediconzoli, perché cultura a buon mercato; ma perciò stesso pessima per chi deve approfondire i problemi dello spirito, della società, della realtà. E pessima non solo mentalmente, ma anche moralmente $\gg^{3}$. «La mentalità massonica - argomentava Croce - semplifica tutto: la storia che è complicata, la filosofia che è difficile, la scienza che non si presta a conclusioni recise, la morale che è ricca di contrasti e di ansie. Essa passa su tutte queste cose trionfalmente, in nome della ragione, della libertà, della umanità, della fratellanza, della tolleranza» ${ }^{4}$.

2 Cfr. T. Tomasi, Massoneria e scuola dall'Unità ai nostri giorni, Vallecchi, Firenze 1980; F. Conti, Massoneria, scuola e questione educativa nell'Italia liberale, «Annali di storia dell'educazione e delle istituzioni scolastiche», 11, 2004, pp. 11-27; A.M. Isastia, A. Visani, L'idea laica tra Chiesa e massoneria. La questione della scuola, Atanòr, Roma 2008.

3 B. Croce, La «mentalità massonica», in Id., Cultura e vita morale. Intermezzi polemici, Bibliopolis, Napoli 1993 (prima ed. 1914), p. 143.

4 Ibidem. 
L'illustre studioso abruzzese sarebbe rimasto probabilmente stupito nel vedere quanti scienziati e fior di intellettuali frequentavano in quegli anni le logge massoniche italiane. Il Grande Oriente seppe infatti attrarre non soltanto esponenti di quel ceto medio imbevuto di positivismo a buon mercato e di «anticlericalismo parolaio $\gg^{5}$, che suscitava il disprezzo di Croce, ma anche numerosi accademici, molti dei quali di primissimo rango nelle rispettive discipline. Che ruolo ebbero all'interno delle logge? Come interpretarono il loro massonismo nella sfera professionale e nella vita pubblica?

Dal punto di vista metodologico - vale per i docenti universitari come per ogni altra categoria sociale e professionale - una questione di assoluta rilevanza riguarda proprio la natura di questo vincolo. Le fonti a mia disposizione offrono riscontri inequivocabili sulla loro effettiva affiliazione alle logge massoniche, ma nella maggior parte dei casi sono piuttosto reticenti sulla durata di tale affiliazione. La fonte principale è costituita dai libri matricolari degli iscritti al Goi, dai quali si ricavano notizie sulla data di iniziazione al grado di apprendista e poi sugli avanzamenti ai due gradi successivi di compagno e di maestro. Un percorso che in genere si esauriva nell'arco di uno o due anni. Questi registri sono perlopiù privi di ragguagli sulla successiva permanenza degli affiliati all'interno dell' istituzione. Tali notizie si potrebbero desumere dalla corrispondenza o dai verbali delle singole logge, che però per il periodo dall'Unità al fascismo sono andati in larga parte perduti o distrutti. Qualche rapsodico riferimento si ottiene perciò soltanto dalla consultazione della stampa periodica o da altra documentazione d'archivio. Il risultato, ripeto, è che per la grande maggioranza degli individui di cui si parlerà in questo contributo possiamo dire con certezza che in un certo momento della loro vita essi furono iniziati alla massoneria, ma non sappiamo se questa loro adesione si protrasse per pochi anni oppure per un arco di tempo molto più lungo.

I massoni ritengono che la qualifica di 'fratello', una volta avvenuta l'iniziazione e acquisito il grado di maestro, si mantenga per tutta la vita e che un libero muratore possa tuttalpiù cessare di partecipare attivamente ai lavori di loggia, mettendosi 'in sonno', senza tuttavia perdere le prerogative di massone e conservando un legame stretto con l'istituzione. Del resto, quello che egli stringe entrando in massoneria è un vincolo di fratellanza, è la scelta di far parte di un sodalizio che distingue nettamente i profani dagli affiliati, ai quali impone precise norme di comportamento, insegna come riconoscere i fratelli e come soccorrerli in caso di bisogno ${ }^{6}$. Insomma: semel abbas, semper abbas. In realtà, almeno nel periodo in questione le cose non andarono esattamente così. Per molti individui, compresi i professori universitari, l'adesione alla massoneria è da equipa-

Ivi, p. 144.

Cfr. F. Conti, I fratelli e i profani. La massoneria e l'idea di fratellanza fra Sette e Ottocento, in G. Bertrand, C. Brice e G. Montègre (a cura di), Fraternité. Pour une histoire du concept, CRHIPA (Centre de recherche en histoire et histoire de l'art. Italie, Pays alpins), Grenoble 2012, pp. 137-155. Per uno sguardo più generale si veda P.-Y. Beaurepaire, L'autre et le frère. L'étrangers et la franc-maçonnerie en France au XVIII siècle, Honoré Champion, Paris 1998. 
rare a quella che essi fecero ad altre associazioni di tipo politico o culturale. Fu motivata da ragioni ideali, dall'identificazione con il progetto dell'istituzione liberomuratoria (la laicità dello Stato, il mito del progresso scientifico, la modernizzazione civile e sociale del Paese), oppure da legami familiari e professionali, o ancora da convenienze e ragionamenti di altra natura. Ma proprio per questo ebbe in genere una durata limitata, si collocò in una fase circoscritta della loro esistenza. In taluni casi fu seguita da una presa di distanza o addirittura dal ripudio di quella esperienza. Più spesso seguì il destino di tanti vincoli associativi similari, che dopo un certo arco di tempo, una volta esaurite le ragioni ideali o pratiche che li avevano originati, finiscono con l'interrompersi.

Certo è che per tutta l'età liberale l'adesione alla massoneria, anche qualora fosse stata di breve durata, rappresentò una scelta impegnativa, consapevole, mai banale. Essa ci rivela tratti importanti degli orientamenti politici e culturali, della mentalità, del carattere degli uomini che presero tale decisione, attraverso la quale entrarono a far parte di un network relazionale esteso su tutto il territorio italiano e con ramificazioni all'estero. Una struttura associativa che vantava fra i suoi affiliati molti esponenti dell'establishment politico e istituzionale e una consistente rappresentanza dei vari notabilati locali, ma che al tempo stesso suscitava profondi sentimenti di avversione in vasti e diversificati segmenti della società (dai cattolici ai socialisti, ai nazionalisti) ${ }^{7}$.

Un'ultima doverosa considerazione, con riferimento a quanto specificamente interessa in questo contributo, riguarda il fatto che nel novembre 1925, quando fu promulgata la legge contro le associazioni segrete, le due principali obbedienze massoniche attive in quel momento nella Penisola, il Grande Oriente d'Italia di Palazzo Giustiniani e la Gran Loggia d'Italia di Piazza del Gesù, furono obbligate allo scioglimento. Quello fu l'atto conclusivo di una strategia di forte contrapposizione fra il fascismo e la massoneria, che era iniziata nel 1923 e aveva completamente rovesciato la relazione simpatetica instauratasi in precedenza ${ }^{8}$. Il 13 febbraio 1923 il Gran consiglio del fascismo stabilì infatti l'incompatibilità fra l'appartenenza al Pnf e alla massoneria, decisione che creò i presupposti per la fusione, avvenuta qualche giorno più tardi, fra il partito nazionalista e quello fascista. Da allora il fascismo fece proprio tutto l'antico livore antimassonico dei nazionalisti e si attestò su una linea di netto conflitto con Palazzo Giustinia$\mathrm{ni}$, che per qualche tempo ancora risparmiò invece l'obbedienza di Piazza del Gesù. Da qui all'avvio di una feroce campagna antimassonica, che trovò ampia ospitalità sulla stampa, il passo fu breve. Le devastazioni e gli assalti squadristici subiti dalle logge fra il 1923 e il 1925 ne furono l'immediato corollario'.

Cfr. F. Conti, Storia della massoneria italiana. Dal Risorgimento al fascismo, il Mulino, Bologna 2003.

8 Cfr. G. Padulo, L'ingrata progenie. Grande guerra, Massoneria e origini del fascismo (19141923), Nuova immagine editrice, Siena 2018.

9 Su questa fase della storia della massoneria italiana e per ulteriori riferimenti bibliografici cfr. F. Conti, Massoneria e fascismo: dalla marcia su Roma alla legge sulle associazioni segre- 
La conseguenza fu che dal 1925 la massoneria in Italia venne messa al bando e non vi furono logge in attività fino all'indomani del 25 luglio 1943. Alcuni dirigenti della massoneria furono arrestati e condannati al carcere o al confino, altri scelsero la via dell'esilio ${ }^{10}$. La maggior parte di essi si mise «in sonno», pose fine cioè a ogni attività di tipo iniziatico. E non si ebbero, ovviamente, nuove affiliazioni. Tutti gli accademici di ambito giuridico ed economico di cui si parlerà nelle pagine che seguono fecero dunque il loro ingresso in massoneria nel periodo precedente al 1925 .

\section{I giuristi massoni}

Attingendo a varie fonti (i libri matricolari del Goi, alcuni repertori biografici di massoni illustri ${ }^{11}$, studi di ambito locale), ho individuato un elenco di 161 docenti universitari la cui affiliazione alla massoneria, da una serie di riscontri incrociati dei quali non è qui possibile dar conto in dettaglio, può considerarsi certa. Tale affiliazione avvenne, nella quasi totalità dei casi, fra il 1860 e il 1924. Come si vede, l'arco temporale di riferimento è assai lungo, ma se consideriamo il numero dei professori universitari allora in attività - all'inizio del Novecento erano «poco più di $1100 \gg^{12}-$ il campione preso in esame non appare poi così esiguo. E soprattutto la rilevanza scientifica, sociale e politica di molti dei personaggi compresi in questo elenco fa sì che dall'analisi del loro percorso biografico e accademico si possano trarre annotazioni di un certo interesse.

Se guardiamo a come si suddividevano fra i grandi macro-settori disciplinari emerge che oltre un terzo erano medici e veterinari (55, il 34,2\% del totale); poco meno di un quarto (37, pari al 23\%) erano letterati, filosofi, storici, archeologi, umanisti; 27 erano scienziati (16,8\%); 25 erano giuristi (15,5\%); 14 erano docenti di economia e di statistica $(8,7 \%) ; 3$ erano professori di ingegneria o architettura $(1,8 \%)$. Ciò che rivelano immediatamente queste cifre è la capacità da parte della massoneria italiana postunitaria di attrarre iscritti in tutti gli ambiti scientifici, anche se talvolta i confini fra l'uno e l'altro erano assai labili. Per esempio, ho inserito fra i giuristi Giovanni Bovio, che fu principalmente un filosofo, in ragione del fatto che insegnò filosofia del diritto e diritto pubblico

te, in Id. (a cura di), La massoneria italiana da Giolitti a Mussolini. Il gran maestro Domizio Torrigiani, Viella, Roma 2014, pp. 85-108.

10 Cfr. S. Fedele, La massoneria italiana nell'esilio e nella clandestinità, 1927-1939, Franco Angeli, Milano 2005.

11 Il principale è quello di V. Gnocchini, L'Italia dei liberi muratori. Piccole biografie di massoni famosi, Erasmo-Mimesis, Roma-Milano 2005. Gnocchini è stato per molti anni 'grande archivista' del Goi e l'appartenenza alla massoneria dei personaggi riportati nel suo elenco, tranne poche eccezioni, è in genere suffragata da solidi riscontri documentari.

12 M. Moretti, $L$ '«Associazione Nazionale fra i Professori Universitari $i$ e la politica universitaria nell'età giolittiana. Note ed osservazioni, in A. Romano (a cura di), Università in Europa. Le istituzioni universitarie dal Medio Evo ai nostri giorni: strutture, organizzazione, funzionamento, Rubbettino, Soveria Mannelli 1995, p. 589. 
comparato all'Università di Napoli. Lo stesso può dirsi per Angelo Camillo De Meis, anch'egli filosofo e scienziato, che qui è stato inserito nella categoria dei medici perché fu professore di fisiologia a Modena e poi di storia della medicina a Bologna. Oppure si pensi ancora a Quirico Filopanti, pseudonimo dello scrittore e patriota Giuseppe Barilli, qui compreso fra gli scienziati poiché nella sua travagliata esperienza accademica fu docente di meccanica applicata all'Università di Bologna.

I giuristi e gli economisti, sommati insieme, rappresentavano dunque quasi un quarto del totale, per l'esattezza il 24,2\%. Meno dei medici, degli umanisti e degli scienziati, ma pur sempre una porzione ragguardevole dell'universo massonico. E va ricordato che qui si sono presi in considerazione soltanto coloro che ebbero a vario titolo incarichi di docenza universitaria. Tutt'altro riscontro si avrebbe qualora s'includessero altre figure del mondo giuridico, come per esempio gli avvocati, che furono numerosissimi nelle logge massoniche dell'Italia liberale come pure di quella repubblicana. Dei venticinque giuristi censiti nel mio elenco quelli ancora attivi, o comunque viventi, nel periodo fra le due guerre furono sedici. Ne riporto i nomi in ordine alfabetico facendoli seguire da alcune sommarie notizie biografiche relative soprattutto alla loro carriera accademica e alle eventuali cariche politiche e amministrative ricoperte. Ometto di appesantire le note con eccessivi riferimenti bibliografici e per ciascuno di essi mi limito a indicare $\mathrm{i}$ contributi di studio più significativi:

1) Agostino Berenini (1858-1939). Professore di diritto e procedura penale a Sassari dal 1900, nel 1902 approdò all'Università di Parma, di cui fu rettore dal 1919 al 1925. A Parma fu presidente del Consiglio provinciale e del Consiglio dell'Ordine degli avvocati. Deputato socialista dal 1892, nel 1912 seguì Bissolati nel Partito socialista riformista. Fu Ministro della pubblica istruzione nel governo Orlando dall'ottobre 1917 al giugno $1919^{13}$. Non si sa con precisione quando fu iniziato massone. All'inizio del Novecento risultava però membro influente del Grande Oriente d'Italia e fu coinvolto in importanti commissioni dal gran maestro Ernesto Nathan nel 1901 e dal suo successore Ettore Ferrari nel $1912^{14}$.

2) Francesco D’Alessio (1886-1949). Dopo aver tenuto un corso libero di diritto amministrativo nell'Università di Roma nell'anno accademico 1912-1913, nel febbraio $1914 \mathrm{fu}$ nominato professore straordinario nella libera Università

13 Fra le sue opere Offese e difese. Appunti di diritto criminale, Tip. L. Battei, Parma 1887; Limiti della prova in criminale, Tip. L. Battei, Parma 1887; Azione ed istruzione penale. Organi e funzioni. Saggio critico, Tip. L. Battei, Parma 1888; Teoria delle pene, Stab. Tip. E. Reggiani, Milano 1889; Lezioni di procedura penale, Lit. Bartoli, Parma 1900; Commentario al nuovo codice di procedura penale, direttori avv. R. Garofalo, proff. A. Berenini, A. Zerboglio, E. Florian, Vallardi, Milano 1913-1914.

14 Cfr. Gnocchini, L'Italia dei liberi muratori, cit., s.v. e M. Novarino, Compagni e liberi muratori. Socialismo e massoneria dalla nascita del Psi alla grande guerra, Rubbettino, Soveria Mannelli 2015, pp. 77-78. 
di Urbino e poi si trasferì in quella di Macerata, dove insegnò fino al novembre 1922. Nel 1919 fu eletto deputato nel collegio di Potenza e riconfermato nel 1921 e nel 1924, l'ultima volta in una lista fiancheggiatrice di quella fascista. Alla fine del 1924 D'Alessio, che nel frattempo era stato chiamato a insegnare diritto amministrativo e scienza dell'amministrazione prima a Cagliari e poi a Pavia, entrò a far parte del comitato di redazione della «Rivista di diritto pubblico». Iscrittosi al Partito nazionale fascista, nel luglio 1925 fu chiamato da Volpi a ricoprire il posto di sottosegretario al Ministero delle finanze, dove legò il suo nome alla riforma della finanza locale varata nell'ottobre di quell'anno. La sua rapida ascesa nella gerarchia fascista (nel marzo 1926 fu eletto segretario federale del Pnf di Potenza) s'interruppe bruscamente sul finire degli anni Venti, quando gli vennero rimproverate le relazioni di amicizia con alcuni ex parlamentari antifascisti e nel $1931 \mathrm{gli}$ fu addirittura ritirata la tessera del Pnf. Nel 1935 si trasferì all'Università di Napoli per assumervi la cattedra di istituzioni di diritto pubblico, da cui nel 1936 passò a quella di diritto finanziario e scienza delle finanze per tornare finalmente nel 1938 alla sua antica disciplina del diritto amministrativo. Dopo la caduta del fascismo fu sospeso dall'ufficio di professore in base al Decreto legge sull'epurazione del luglio 1944 ed egli stesso preferì chiedere il collocamento a riposo. Tuttavia, una sentenza della Corte d'appello di Roma del 1946 e un successivo decreto del presidente del Consiglio del dicembre 1947 annullarono il precedente giudizio e gli consentirono di riprendere servizio presso l'Università di Napoli ${ }^{15}$.

D'Alessio ebbe una militanza massonica relativamente lunga. Iniziato il 28 ottobre 1910 nella Loggia Peucetia di Bari, nei primi anni Venti, quando era deputato, risultava affiliato alla Loggia Rienzi di Roma ${ }^{16}$.

3) Alfredo De Marsico (1888-1985). Libero docente in diritto e procedura penale nella Università di Roma dal maggio 1915, nel 1922 vinse la cattedra nella stessa disciplina a Camerino. Successivamente la ottenne a Cagliari (marzo 1926), Bari (novembre 1926), Bologna (novembre 1931), Napoli (1935). Dall'ottobre 1935 divenne ordinario di diritto processuale penale, e dal giugno 1940 ordinario di diritto penale nell'Università di Roma, dove insegnò, con la sospensione dovuta all'epurazione, fino al 1958. Inserito nel listone nazional-fascista, nel 1924 fu eletto deputato per la circoscrizione di Napoli e poi confermato nelle elezioni plebiscitarie del 1929 e 1934. Nel 1939 divenne consigliere nazionale

15 Fra le sue opere Rapporti e conflitti fra le due sezioni giurisdizionali del Consiglio di Stato, Società editrice libraria, Milano 1912; Le parti nel giudizio amministrativo, Società editrice libraria, Milano 1915; Dalla dichiarazione dei diritti dell'uomo al moderno stato di diritto, Stab. cromo-tipografico, Roma 1915; Istituzioni di diritto amministrativo italiano, Utet, Torino 1932-1934; Commento del Testo Unico della Legge comunale e provinciale 3 marzo 1934, n. 383, Utet, Torino 1936; Corso di diritto finanziario, Jovene, Napoli 1937; Le leggi sulla giustizia amministrativa commentate, Utet, Torino 1938.

16 Cfr. Archivio storico del Grande Oriente d'Italia, Roma, Libri matricolari degli iscritti (d'ora in poi Asgoi, Matricola) e Gnocchini, L'Italia dei liberi muratori, cit., s.v. 
della Camera dei fasci e delle corporazioni in rappresentanza degli avvocati e procuratori (oltre che docente universitario fu infatti un celebre penalista e nel secondo dopoguerra a lungo presidente dell'Ordine degli avvocati di Napoli). Nel febbraio $1943 \mathrm{fu}$ nominato Ministro di grazia e giustizia da Mussolini, contro il quale però votò il 25 luglio successivo nella famosa seduta del Gran consiglio del fascismo, quando fu uno degli estensori dell'ordine del giorno Grandi. Condannato a morte in contumacia dalla Repubblica sociale italiana nel processo di Verona del gennaio 1944, dopo la caduta del fascismo subì le conseguenze, come detto, dei procedimenti epurativi che lo allontanarono per alcuni anni dall'attività forense e dall'insegnamento universitario. De Marsico tornò poi anche all'impegno politico e nel $1953 \mathrm{fu}$ eletto come indipendente nella lista monarchica di Achille Lauro per la circoscrizione di Avellino-Sala Consilina ${ }^{17}$.

Proprio in una loggia di Sala Consilina, la Giuseppe Mazzini, era stato iniziato alla massoneria il 19 luglio $1911^{18}$.

4) Paolo Greco (1889-1974). Laureatosi nel 1911 a Napoli, sua città natale, nel 1931 divenne professore ordinario di diritto commerciale all'Università di Macerata senza tuttavia abbandonare la professione di avvocato che aveva già intrapreso. Nel 1933 passò a insegnare all'Università di Parma e dal 1935 a quella di Torino, dove si trasferì definitivamente pur mantenendo un incarico anche alla Bocconi, della quale fu anche rettore dal 1938 al 1945. Dopo l'8 settembre 1943 entrò a far parte del Comitato di liberazione nazionale piemontese in rappresentanza del Partito liberale, assumendone la presidenza che tenne fino al 1945. Dopo la guerra tornò all'insegnamento e all'esercizio della professione forense, pubblicando numerosi saggi, diversi dei quali dedicati al diritto d'autore e all'impresa. Nel 1952 fu tra i fondatori dell'Istituto universitario di studi europei ${ }^{19}$.

Fu iniziato nella Loggia I costanti nella fede italica di Napoli il 18 luglio $1915^{20}$.

17 Fra le sue opere La rappresentanza nel diritto processuale penale, Società editrice libraria, Milano 1915; Studi di diritto penale, Morano, Napoli 1930; Coscienza e volontà nella nozione del dolo, Morano, Napoli 1930; La riforma della legislazione, Mondadori, Milano 1934; Diritto penale, Jovene, Napoli 1935; Lezioni di diritto processuale penale, Jovene, Napoli 1936; Legislazione e giustizia nel fascismo, Mondadori, Milano 1939; Nuovi studi di diritto penale, Edizioni scientifiche italiane, Napoli 1951; Lo Stato nella difesa dalla violenza (discorsi escritti 1961-1974), Volpe, Roma 1982.

18 Cfr. Asgoi, Matricola.

19 Fra le sue opere Trasporto marittimo e noleggio, F. Sangiovanni \& figlio, Napoli 1921; Delegazione e obbligazione nel diritto civile italiano, Libr. Detchen e Rocholl, Napoli 1928; Il contratto collettivo di lavoro, Ed. diritto del lavoro, Roma 1929; Lezioni di diritto commerciale, Tip. A. Zanlari \& C., Parma 1934; Corso di diritto bancario, Cedam, Padova 1936; Corso di diritto commerciale secondo la nuova codificazione, Giappichelli, Torino 1942; La compravendita e altri contratti, Malfasi, Milano 1947; Corso di diritto commerciale. Impresa, azienda, società, Malfasi, Milano 1948; La società nel sistema legislativo italiano. Lineamenti generali, Giappichelli, Torino 1959.

20 Cfr. Asgoi, Matricola. 
5) Filippo Grispigni (1884-1955). Nato a Viterbo, dal 1923 fu professore di diritto penale a Camerino e successivamente a Cagliari, Milano e Roma. Insieme a Eugenio Florian fu tra gli esponenti maggiori della scuola positiva criminologica italiana. Nel 1942 fu chiamato a Roma, dapprima alla cattedra di procedura penale, poi a quella di diritto penale ${ }^{21}$.

Fu iniziato nella Loggia Nino Bixio di Viterbo il 14 dicembre 1907 e promosso al grado di compagno nel maggio $1909^{22}$.

6) Alessandro Groppali (1874-1959). Studiò a Cremona, dove fu allievo di Arcangelo Ghisleri, quindi a Padova. Si laureò in giurisprudenza e poi in filosofia (1898) con la tesi La genesi sociale del fenomeno scientifico, pubblicata a Torino nel 1899 con la prefazione del suo maestro Roberto Ardigò. Groppali fu incaricato di sociologia all'Università di Ferrara dal 1899 al 1902, finché, divenuto ordinario, insegnò filosofia del diritto e materie affini a Modena dal 1902 al 1924 e a Milano dal 1924 al 1931, continuando, comunque, a esercitare la professione forense. Caduto in disgrazia agli occhi del regime per aver deposto nel 1929 davanti a una commissione d'inchiesta contro il gerarca Roberto Farinacci ed esser stato da questi denunciato per diffamazione, fu trasferito d'ufficio all'Università di Cagliari. Durante la permanenza a Cagliari (1931-1939) divenne preside, carica che gli fu poi revocata perché privato, dopo lo scontro con Farinacci, della tessera del Partito nazionale fascista che aveva preso nel 1919. Nel 1939 ottenne il trasferimento a Venezia e nel 1945, dopo la caduta del regime, venne reintegrato nella sede di Milano, dove insegnò fino al $1949^{23}$.

Ebbe una lunga permanenza nelle file massoniche, essendo stato iniziato il 9 luglio 1902 nella Loggia Nicola Fabrizi - Secura fides di Modena e nel 1918 risultando ancora affiliato alla Loggia Quinto Curzio di Cremona, la stessa a cui era iscritto il futuro ras fascista Roberto Farinacci ${ }^{24}$.

7) Francesco Innamorati (1853-1923). Avvocato e giurista, insegnò per molti anni diritto penale nell'Università di Perugia, dove fu preside della Facoltà di

21 Fra le sue opere La odierna scienza criminale in Italia, Società editrice libraria, Milano 1909; Il nuovo diritto criminale negli avamprogetti della Svizzera, Germania ed Austria, Società editrice libraria, Milano 1911; Introduzione alla sociologia criminale, Utet, Torino 1925; Corso di diritto penale secondo il nuovo codice, Cedam, Padova 1932; Diritto processuale penale, Unione tipografica, Milano 1942.

22 Cfr. Asgoi, Matricola.

23 Fra le sue opere Saggi di sociologia, Battistelli, Milano 1899; Elementi di sociologia, Libreria moderna, Genova 1905; Filosofia del diritto, Hoepli, Milano 1906; Sociologia e filosofia del diritto, Società libraria editrice pontremolese, Piacenza 1908; La sociologia, Sonzogno, Milano 1913; La morale sociale, Giusti, Livorno 1915; Istituzione di scienza generale del diritto, Savoldi, Bergamo 1921; Dottrina generale dello Stato, Giuffrè, Milano 1936; Istituzioni di diritto pubblico, Zanetti, Venezia 1942; Filosofia del diritto, Ambrosiana, Milano 1944; Sociologia e diritto, Ambrosiana, Milano 1945; Avviamento allo studio del diritto, Giuffrè, Milano 1951.

24 Cfr. Asgoi, Matricola. 
giurisprudenza. Nelle elezioni suppletive del 1914 fu eletto nel collegio di Perugia II nelle file dei demo-costituzionali. Alla Camera fece parte della Commissione per la riforma del codice di procedura penale ${ }^{25}$.

Fu iniziato nel 1885 nella Loggia Francesco Guardabassi di Perugia, di cui fu maestro venerabile dal 1894 al 1897. Nel 1915 risultava ancora membro della loggia, nella quale ricopriva la carica di oratore ${ }^{26}$.

8) Silvio Lessona (1887-1969). Seguendo le orme del padre Carlo, ordinario di procedura civile, fu professore universitario dal 1926. Insegnò diritto sanitario e poi diritto pubblico e amministrativo nelle Università di Siena, Firenze e Bologna. A Bologna fondò la Scuola di perfezionamento in scienze amministrative, di cui fu direttore ${ }^{27}$.

Fu iniziato il 10 maggio 1920 nella Loggia Concordia di Firenze, di cui risultava essere uno dei dignitari anche nel 1924. Nel secondo dopoguerra fu ancora un membro attivo della ricostituita loggia Concordia ${ }^{28}$.

9) Arnaldo Lucci (1871-1945). Di origini abruzzesi, si formò presso l'Università di Napoli, dove fu poi libero docente di diritto civile. Avvocato, nel 1913 fu eletto deputato per il Partito socialista e confermato nel 1921 e nel $1924^{29}$.

$\mathrm{Fu}$ affiliato col grado di maestro nella Loggia Losanna di Napoli il 10 marzo $1898^{30}$.

10) Vincenzo Manzini (1872-1957). Laureatosi all'Università di Ferrara e divenuto avvocato penalista, conseguì dapprima la libera docenza in diritto penale e poi nel 1898 la cattedra di diritto e procedura penale (come ordinario dal 1902). Nel corso della carriera accademica insegnò nelle Università di Ferrara, Sassari, Siena, Torino, Pavia, finché nel 1920 si trasferì a Padova, dove tenne

25 Fra le sue opere I nuovi orizzonti del diritto penale e l'antica scuola italiana, Santucci, Perugia 1887; Trattato dell'estinzione e dell'azione penale e delle condanne penali, Vallardi, Milano 1891; Sui delitti contro l'amministrazione della giustizia, Tip. Boncompagni, Perugia 1893.

26 Cfr. Asgoi, Matricola e V. Gnocchini, Logge e massoni in Umbria, a cura di S. Bellezza, Futura edizioni, Perugia 2014.

27 Fra le sue opere Il medico condotto nella legge italiana, Sansoni, Firenze 1912; Trattato di diritto sanitario, 2 voll., Bocca, Torino 1914-1921; I sindacati e lo Stato fascista, Carnesecchi, Firenze 1928; Istituzioni di diritto pubblico, Poligrafica universitaria, Firenze 1930; Introduzione al diritto amministrativo e suoi principi, Editrice universitaria, Firenze 1952; La giustizia amministrativa, Zanichelli, Bologna 1955; Problemi amministrativi della società moderna, Noccioli, Firenze 1967.

28 Cfr. F. Conti, Firenze massonica. Il libro matricola della Loggia Concordia, 1861-1921, Polistampa, Firenze 2012, p. 308 e Id., All'obbedienza di Palazzo Giustiniani: le logge del Grande Oriente d'Italia dalla liberazione agli anni Sessanta, in Id. (a cura di), Logge e liberi muratori a Firenze. Dall'età dei Lumi al secondo Novecento, il Mulino, Bologna 2007, p. 442.

29 Fra le sue opere Il diritto al sottosuolo, De Bonis, Napoli 1898; Giustizia nuova, Nerbini, Firenze 1902; Teoria generale delle obbligazioni nel diritto civile italiano, Pierro, Napoli 1903.

30 Cfr. Asgoi, Matricola. 
per incarico anche gli insegnamenti di storia del diritto italiano e di legislazione del lavoro nella facoltà di Giurisprudenza e di diritto militare presso la facoltà di Scienze politiche. Iscritto dal gennaio $1925 \mathrm{al}$ Partito nazionale fascista, nel 1931 fu eletto preside di Giurisprudenza. Fu anche membro del Consiglio superiore forense, della commissione centrale per gli avvocati e i procuratori, e dal 1935 accademico dei Lincei ${ }^{31}$.

Fu iniziato il 19 febbraio 1904 nella Loggia Giovanni Maria Angioy di Sassari ed elevato a maestro nel giugno $1905^{32}$.

11) Nunzio Nasi (1850-1939). Deputato di Trapani dal 1886, Ministro delle poste nel Gabinetto Pelloux (1898-1899) e dell' istruzione con Zanardelli (19001903), nel $1908 \mathrm{fu}$ condannato a undici mesi di reclusione per peculato. I suoi elettori, ritenendolo ingiustamente perseguitato, lo rimandarono da allora costantemente al parlamento fino al 1924, non tenendo conto dei successivi annullamenti delle elezioni, finché nel 1926 fu dichiarato decaduto insieme con gli altri deputati antifascisti. Nel $1897 \mathrm{fu}$ chiamato quale libero docente di filosofia del diritto prima all'Università di Palermo e poi a quella di Roma ${ }^{33}$.

Affiliato col grado di maestro il 25 settembre 1893 nella Loggia Centrale di Palermo, fu maestro venerabile della Loggia Roma che venne inaugurata nella capitale il 23 dicembre 1900. Dal 1901 al $1902 \mathrm{fu}$ poi presidente della Gran loggia di rito simbolico. Prima che fosse coinvolto nello scandalo che ne segnò la carriera politica e accademica, si parlava di lui come del più autorevole candidato alla successione di Ernesto Nathan nella carica di gran maestro del Grande Oriente d'Italia ${ }^{34}$.

12) Giacomo Perticone (1892-1979). Dal 1925 fu libero docente di filosofia del diritto e dottrina dello Stato all'Università La Sapienza di Roma sotto la guida di Giorgio Del Vecchio. Dal 1927 ebbe poi incarichi universitari, non solo per la filosofia del diritto, ma anche per le materie civilistiche, presso l'Università di Ferrara. Ternato per la cattedra di filosofia del diritto in un concorso bandito nel 1932 dall'Università di Siena, Perticone divenne professore straordinario e quindi ordinario a Ferrara negli anni accademici 1934-1935 e 1935-1936. Nell'ottobre 1936 fu poi trasferito all'Università di Perugia, dove fu per breve tempo preside della facoltà di Giurisprudenza, e dal 1939 all’Università di Pisa.

31 Fra le sue opere, molte delle quali più volte ristampate: Trattato del furto e delle sue varie specie, Utet, Torino 1902; Trattato di diritto penale italiano, Bocca, Torino 1908; Istituzioni di diritto processuale penale, Bocca, Torino 1917; La legislazione penale di guerra, Utet, Torino 1918; Trattato di diritto processuale penale, Bocca, Torino 1924-1925; Diritto penale militare, Cedam, Padova 1928; La superstizione omicida e i sacrifici umani con particolare riguardo alle accuse contro gli ebrei, Cedam, Padova 1930; Codici penali militari, Cedam, Padova 1943.

32 Cfr. Asgoi, Matricola.

33 Fra le sue opere di ambito giuridico La teoria del progresso legislativo, Rizzi, Trapani 1875; Il diritto e la guerra, G. Colitti e figlio, Campobasso 1919.

34 Cfr. Asgoi, Matricola e F. Conti, Storia della massoneria italiana, cit. 
Qui divenne stretto collaboratore di Giuseppe Bottai e della Scuola superiore di scienze corporative. Dopo la guerra Perticone concluse la sua carriera accademica all'Università di Roma presso la facoltà di Scienze politiche, dove insegnò storia dei partiti e dei movimenti politici (1956-1962) ${ }^{35}$.

Fu iniziato nella Loggia Palingenesi di Catania il 30 giugno $1916^{36}$.

13) Luigi Rava (1860-1938). Conseguita nel 1884 la libera docenza di filosofia del diritto, fin dall'anno successivo tenne lezioni presso l'Università di Bologna e dal 1886 anche nell'Ateneo senese. Insegnò inoltre contabilità di Stato sempre a Bologna e in seguito a Pavia, dove nel 1889 vinse il concorso da professore straordinario in filosofia del diritto, cattedra sulla quale rimase fino al 1898. Insegnò poi scienza dell'amministrazione nella facoltà di Giurisprudenza di Bologna, cattedra che tenne (dal 1898 come professore ordinario), con alcune interruzioni dovute ai suoi impegni politici, fino al 1915.

La vera passione di Rava fu tuttavia la politica. Eletto deputato nel 1891, fu vicepresidente della Camera dal 1914 al 1919, sottosegretario nei governi Crispi e Zanardelli, Ministro all'agricoltura, industria e commercio dal 1903 al 1905 negli esecutivi Giolitti e Fortis, Ministro della pubblica istruzione dal 1906 al 1909 ancora con Giolitti. Nel 1914 Rava fu poi per alcuni mesi Ministro delle finanze nel governo Salandra. Nominato senatore nel 1920, nello stesso anno fu eletto sindaco di Roma (lo restò fino al maggio 1921). Dal 1922 al 1934 fece parte della Commissione finanze del Senato e nel 1932 fu nominato ministro di Stato ${ }^{37}$.

Fu regolarizzato maestro nella Loggia Roma di Roma il 9 ottobre 1906. Nel 1914 figurava fra i dirigenti della Gran loggia del rito simbolico italiano ${ }^{38}$.

14) Giuseppe Semmola (1849-1920). Professore di diritto e procedura penale all'Università di Napoli. Eletto deputato nel 1890 per una sola legislatura, fu a lungo consigliere comunale a Napoli ${ }^{39}$.

Intorno al 1873 risultava affiliato alla Loggia La fratellanza di Napoli ${ }^{40}$.

35 Fra le sue opere su tematiche giuridiche La libertà e la legge. Regime politico e ordine giuridico, Anonima romana editoriale, Roma 1936; Linee di una teoria generale del diritto, Giuffrè, Milano 1936; Elementi di una dottrina generale del diritto e dello Stato, Giuffrè, Milano 1939; Lezioni di storia e teoria del diritto internazionale, Edizioni dell'Ateneo, Roma 1949; Il diritto e lo Stato nel pensiero italiano contemporaneo, Cedam, Padova 1950; La filosofia del diritto come filosofia della giustizia, Edizioni dell'Ateneo, Roma 1962; Scritti di filosofia politica e giuridica, Giuffrè, Milano 1969.

36 Cfr. Asgoi, Matricola.

37 Fra le sue opere Filosofia del diritto e definizione del diritto, Zanichelli, Bologna 1885; La filosofia del diritto nel pensiero italiano, Torrini, Siena 1887; La filosofia civile e giuridica in Italia prima della Rivoluzione francese, Vallardi, Milano 1889.

38 Cfr. Asgoi, Matricola.

39 Fra le sue opere Il reato di diffamazione. Studi, De Angelis, Napoli 1884; La censura pubblica nei liberi ordinamenti e la repressione delle ingiurie, Lacava, Napoli 1886; Proemio allo studio di legislazione penale comparata, Pironti, Napoli 1908.

40 Cfr. Gnocchini, L'Italia dei liberi muratori, cit., s.v. 
15) Angelo Sraffa (1865-1937). Nei primi decenni del Novecento si segnalò per gli innovativi studi di diritto societario, fallimentare, della concorrenza e in materia di lavoro, tutti connotati da una peculiare sensibilità per i fenomeni economici. Avvocato di successo, fu professore di diritto commerciale in varie università (Macerata, Messina, Parma, Torino, Milano) e fin dal 1902 insegnò anche alla Bocconi, di cui fu rettore dal 1917 al 1926. Nel 1903 fondò con Cesare Vivante la «Rivista del diritto commerciale». Nel primo dopoguerra partecipò alle commissioni legislative per la riforma del codice di commercio ${ }^{41}$.

Il 9 dicembre $1893 \mathrm{fu}$ affiliato col grado di maestro nella Loggia Carlo Darwin di Pisa ${ }^{42}$.

16) Cino Vitta (1873-1956). Fu professore di diritto amministrativo all'Istituto di scienze sociali di Firenze e poi nelle Università di Cagliari, Modena e Torino. Insegnò anche diritto internazionale e tenne conferenze all'Accademia dell'Aja nel 1930, 1934 e $1936^{43}$.

Fu affiliato col grado di maestro nella Loggia Concordia di Firenze il $1^{\circ}$ luglio $1903^{44}$.

Come emerge da queste brevi note bio-bibliografiche, l'elenco comprende nomi importanti della cultura giuridica italiana fra le due guerre. Molti di essi ricoprirono incarichi direttivi nei rispettivi atenei: furono rettori, presidi, direttori di istituto. Assai numerosi furono anche coloro che all'attività d'insegnamento e di studio affiancarono un intenso impegno politico sia a livello locale che nazionale. Fra i nomi qui segnalati figurano deputati, senatori, ministri oltre che consiglieri comunali e provinciali. Ė opportuno notare che essi, nonostante l'impronta omologante del regime mussoliniano, si distribuirono tra forze politiche di orientamento diverso. Prima e dopo l'avvento del fascismo si schierarono fra i liberali di varia gradazione, i socialisti riformisti, i repubblicani e i monarchici. A conferma del fatto che la massoneria, di cui probabilmente fecero parte soltanto per un certo periodo della loro vita, non s'identificava con un preciso e ben determinato partito politico. Si configurava tutt'al più come un luogo di agglutinamento di élites modernizzanti e progressiste, caratterizzate da una spiccata matrice laica e anticlericale, ma unite altresì da un forte sentimento patriottico che dalla guerra di Libia e dalla campagna interventista del 1914-1915 si

${ }^{41}$ Fra le sue opere Studi di diritto commerciale, Spoerri, Pisa 1891; La liquidazione delle società commerciali, Pellas, Firenze 1891. Su di lui si veda A. Monti, Angelo Sraffa. Un «antiretorico» del diritto, Egea, Milano 2011.

42 Cfr. Asgoi, Matricola.

43 Fra le sue opere Giustizia amministrativa, Hoepli, Milano 1903; Il potere disciplinare sugli impiegati pubblici, Società editrice libraria, Milano 1913; Gli atti collegiali. Principi sul funzionamento dei consessi pubblici con riferimenti alle assemblee private, Athenaeum, Roma 1920; Diritto amministrativo, Utet, Torino 1933; Introduzione allo studio delle scienze giuridiche e istituzioni di diritto pubblico, Giappichelli, Torino 1933.

44 Cfr. Asgoi, Matricola. 
era notevolmente irrobustito fino a non essere più molto distinguibile da quello dei nazionalisti. Fu su questo terreno che maturò l'incontro col primo fascismo e ne nacque la fascinazione di molti massoni per Mussolini, poi frustrata, come si è detto, dalla svolta del 1923, dalla dichiarazione d'incompatibilità tra fascismo e massoneria e dall'inizio della campagna repressiva culminata nella legge contro le associazioni segrete del novembre 1925.

\section{Gli economisti e statistici massoni}

Quanto ai docenti universitari di economia o statistica affiliati alla massoneria quelli presenti nel mio campione sono undici. Eccone l'elenco:

1) Celestino Arena (1890-1967). Laureatosi in Giurisprudenza all'Università di Napoli, trovò dapprima impiego presso l'Amministrazione delle poste $\mathrm{e}$ poi dal 1924 al Ministero degli esteri come consigliere di emigrazione. Dal 1926 fu segretario del Consiglio superiore dell'emigrazione e direttore del «Bollettino dell'emigrazione». Nel frattempo indirizzò i suoi interessi di ricerca verso il diritto del lavoro, la previdenza sociale e infine la politica economica e finanziaria, pubblicando varie opere che dal 1928 gli dischiusero la carriera universitaria a Pisa, dove insegnò legislazione comparata del lavoro e dell'economia e poi politica economica e del lavoro. A Pisa fu uno degli animatori della Scuola di perfezionamento in studi corporativi e collaborò alla rivista «Archivio di studi corporativi» fondata nel 1930 da Bottai. Insieme a Bottai negli anni Trenta diresse la "Nuova collana di economisti stranieri e italiani" che uscì per i tipi della Utet. Nel 1934 fu chiamato come professore straordinario di diritto del lavoro all'Università di Camerino, ma l'anno seguente ottenne di poter tornare a Pisa. Nel 1938 conseguì l'ordinariato e passò a insegnare scienza delle finanze e diritto finanziario a Napoli, dove restò fino al 1942, quando si trasferì a Roma. Dal 1940 al $1945 \mathrm{fu}$ segretario generale dell'Istituto nazionale di finanza pubblica presso la Banca d'Italia. Dopo la guerra fu membro del Consiglio superiore della pubblica istruzione, del Cnel e di numerosi altri organismi italiani e internazionali ${ }^{45}$.

$\mathrm{Fu}$ iniziato nella Loggia Bruzia-Pietro De Roberto di Cosenza il 15 ottobre $1915^{46}$.

2) Gino Arias (1879-1940). Laureatosi in Giurisprudenza a Bologna, fu inizialmente libero docente di storia del diritto italiano. Passato a occuparsi di discipline economiche, dal $1909 \mathrm{fu}$ docente di economia politica a Genova e dal

45 Fra le sue opere La nuova politica economica della Russia, La Società per Azioni, Roma 1923; La dinamica del sistema corporativo, Tip. Pacini Mariotti, Pisa 1933; Corso di economia del lavoro, Cedam, Padova 1933; Scienza delle finanze e diritto finanziario, Jovene, Napoli 1941; Teoria generale della finanza pubblica, Jovene, Napoli 1945; Principi di economia politica e nozioni di statistica, Paravia, Torino 1956.

46 Cfr. Asgoi, Matricola. 
1924 a Firenze, come ordinario di scienza e tecnica del commercio e poi di economia politica. Dal 1930 al 1936, prima del suo trasferimento all’Università di Roma, fu preside della facoltà fiorentina di Economia. Convinto sostenitore del regime fascista, dal 1931 fece parte del Consiglio nazionale delle corporazioni e dal 1934 della Camera dei deputati. Ciò non valse a risparmiarlo dalle leggi razziali che nel 1938 lo costrinsero, in quanto ebreo pur convertito al cattolicesimo, a emigrare in Argentina, dove insegnò economia politica all'Università di Cordoba ${ }^{47}$.

Fu iniziato nella Loggia Lira e Spada di Roma il 22 gennaio $1906^{48}$.

3) Alberto Beneduce (1877-1944). Laureatosi in discipline matematiche a Napoli nel 1902, entrò nel 1904 alla sezione statistica del Ministero di agricoltura, industria e commercio. Nel 1910 conseguì la libera docenza per l'insegnamento di statistica e demografia e l'anno seguente fu chiamato da Nitti a collaborare al progetto di fondazione dell'Istituto nazionale delle assicurazioni, di cui nel 1916 divenne consigliere delegato. Nel frattempo, nel 1914, aveva ottenuto la cattedra di statistica a Genova. Nel 1919 fu eletto deputato per il Partito socialista-riformista e nel 1921 divenne Ministro del lavoro e della previdenza sociale nel governo Bonomi. Da allora si consacrò a una prestigiosa carriera di grand commis d'État che non ebbe soluzioni di continuità con l'avvento del fascismo. Dal 1919 fu a capo del Consorzio di credito per le opere pubbliche (Crediop), dal 1924 dell'Istituto di credito per le imprese di pubblica utilità (Icipu) e dal 1926 della Società per le strade ferrate meridionali (Bastogi). Dal 1933 fu presidente dell'Istituto per la ricostruzione industriale (Iri) e nel $1939 \mathrm{fu}$ nominato senatore ${ }^{49}$.

Iniziato l'11 agosto 1905 nella Loggia Giovanni Bovio di Roma, ebbe un lungo cursus massonico che lo portò a ricoprire importanti cariche direttive nel Grande Oriente d'Italia fino ai primi anni Venti ${ }^{50}$.

4) Luigi (Gino) Borgatta (1888-1949). Allievo di Luigi Einaudi e di Vilfredo Pareto, dal 1915 al 1916 fu professore di politica economica presso l'Istituto superiore di scienze economiche a Venezia e dal 1916 al 1917 presso l'Università

47 Fra le sue opere I trattati commerciali della Repubblica fiorentina, Succ. Le Monnier, Firenze 1901; Le istituzioni giuridiche medievali nella Divina Commedia, Lumachi, Firenze 1901; Il sistema della costituzione economica e sociale italiana nell'età dei Comuni, Roux e Viarengo, Torino-Roma 1905; Principi di economia commerciale, Società editrice libraria, Milano 1917; La questione meridionale, Zanichelli, Bologna 1921; Economia italiana. Scritti di politica economica nazionale, Zanichelli, Bologna 1926; L'economia nazionale corporativa, Libreria del Littorio, Roma 1929; Corso di economia politica corporativa, Soc. ed. Foro italiano, Roma 1936; P. Roggi (a cura di), Antologia di scritti, Fondazione Spadolini-Nuova Antologia, Firenze 2009. Su di lui cfr. O. Ottonelli, Gino Arias (1879-1940). Dalla storia delle istituzioni al corporativismo fascista, Firenze University Press, Firenze 2013.

48 Cfr. Asgoi, Matricola.

49 Cfr. M. Franzinelli, M. Magnani, Beneduce. Il finanziere di Mussolini, Mondadori, Milano 2009.

50 Cfr. Asgoi, Matricola. 
di Sassari. Dal 1920 al 1922 insegnò politica e legislazione doganale nell'Istituto superiore di scienza economica di Torino e scienza delle finanze dal 1923 al 1927 nell'Università di Pisa. Dal 1927 fu titolare della cattedra di diritto finanziario e di scienza delle finanze presso l'Università di Milano e incaricato alla Bocconi ${ }^{51}$.

Fu iniziato nella Loggia Ausonia di Torino il 16 giugno 1909, dove nel 1919 conseguì il grado di maestro ${ }^{52}$.

5) Epicarmo Corbino (1890-1984). Docente di politica commerciale e legislazione doganale presso l'Istituto superiore di scienze economiche di Napoli dal 1923, fu Ministro dell'industria e commercio nei governi Badoglio (1944) e Ministro del tesoro nei primi due governi De Gasperi (1945-1946). Esponente del partito liberale, fu membro della Consulta nazionale, dell'Assemblea costituente e deputato nella prima legislatura. Fu inoltre presidente del Banco di Napoli (1959-65) e socio nazionale dei Lincei dal $1963^{53}$.

Fu iniziato il 22 luglio 1910 nella Loggia Xifonia di Augusta ed elevato al grado di maestro nel $1915^{54}$.

6) Massimo Nino (Natale) Fovel (1880-1941). Fin da giovane militò nel Partito radicale, riconoscendosi nella corrente radical-socialista, e collaborò a vari giornali e riviste con articoli in materie economiche e finanziarie. Nel 1919 aderì al Partito socialista militando nella frazione massimalista e nel 1920 assunse la direzione del «Lavoratore», quotidiano di Trieste, organo ufficiale della Federazione socialista della Venezia Giulia. Docente di scienza delle finanze all'U-

51 Fra le sue opere Che cos'è e cosa costa il protezionismo in Italia. Manualetto antiprotezionista, Libreria della Voce, Firenze 1914; L'economia dinamica, Utet, Torino 1915; Politica commerciale e legislazione doganale, La Litotipo, Padova 1917; Saggi di finanza teoretica, Athenaeum, Roma 1920; Crisi bellica e crisi postbellica, Tip. Baravalle e Falconieri, Torino 1921; Saggi e rassegne critiche (1918-1921), Sten, Torino 1922; La stabilizzazione dei cambi e la bilancia dei pagamenti, Stab. poligrafico dello Stato, Roma 1928; Appunti di scienza delle finanze e diritto finanziario, Giuffrè, Milano 1933; Le finanze pubbliche nell'ultimo decennio, Cedam, Milano 1940; La finanza della guerra e del dopoguerra, Gazzotti, Alessandria 1946.

52 Cfr. Asgoi, Matricola.

53 Fra le sue opere Liberalismo e protezionismo, Cavanna, Pontremoli 1922; I porti marittimi italiani, Tip. Leonardo da Vinci, Città di Castello 1924; Economia dei trasporti marittimi, Tip. Leonardo da Vinci, Città di Castello 1926; Annali dell'economia italiana, 5 voll., Tip. Leonardo da Vinci, Città di Castello 1931-1938; La battaglia dello Jutland vista da un economista, Colombi, Milano 1935; Corso di politica economica e finanziaria, Giuffrè, Milano 1942; Ricostruzione. Scritti e discorsi di un liberale, Giuffrè, Milano 1945; L'economia italiana dal 1860 al 1960, Zanichelli, Bologna 1962; Cronache economiche e politiche, 1946-1973, 4 voll., Istituto editoriale del Mezzogiorno poi Edizioni scientifiche italiane, Torino poi Napoli 1964-1974.

54 Cfr. Asgoi, Matricola. Su di lui cfr. inoltre F. Conti, Massoneria e liberali nel secondo dopoguerra (1943-1958), in G. Berti, E. Capozzi e P. Craveri (a cura di), I liberali italiani dall'antifascismo alla Repubblica, vol. II, Rubbettino, Soveria Mannelli 2010, pp. 547-549. 
niversità di Bologna e poi di diritto commerciale a Ferrara, approdò più tardi al fascismo e divenne uno dei più acuti ideologi dell'economia corporativa ${ }^{55}$.

Fu iniziato nella Loggia Ça ira di Bologna il 7 aprile $1911^{56}$.

7) Arturo Labriola (1873-1959). Socialista, fu tra i leader del sindacalismo rivoluzionario. Dopo aver ripiegato su posizioni più moderate, nel 1913 entrò alla Camera come socialista indipendente e fu deputato per quattro legislature. Interventista nel 1915, fu poi Ministro del lavoro nell'ultimo governo Giolitti (1920-1921). Vivace studioso di storia delle dottrine economiche, nel 1926 ottenne la cattedra di economia nell'Università di Messina, ma ne fu allontanato dal governo fascista e dovette emigrare in Francia e in Belgio, dove insegnò all'Istituto di alti studî di Bruxelles. Rientrato in Italia nel 1936, visse appartato fino alla caduta del fascismo. Reintegrato nella cattedra, fece quindi parte della Consulta nazionale e della Costituente e fu senatore di diritto nella prima legislatura repubblicana ${ }^{57}$.

Iniziato nella Loggia Propaganda massonica di Roma l'11 febbraio 1914, fu gran maestro del Grande Oriente d'Italia in esilio dal giugno 1930 al novembre 1931. Dopo la caduta del fascismo fu a capo di gruppi massonici del rito scozzese che tentarono di rinverdire l'esperienza della Gran Loggia d'Italia di Piazza del Gesù ${ }^{58}$.

8) Vittorio Racca (1876-1957), laureatosi all'Università di Modena, collaborò al «Giornale degli economisti» e fu per qualche tempo assistente di Vilfredo Pareto all'Università di Losanna, dove ebbe un incarico di scienza delle finanze e legislazione sociale. Insegnò poi economia politica all'Università di Roma ${ }^{59}$.

55 Fra le sue opere La colonizzazione interna. Saggio, Tip. Garagnani, Bologna 1906; Scienza politica e scienza dell'amministrazione, Zanichelli, Bologna 1906; Il credito agrario in Italia, Zanichelli, Bologna 1909; Democrazia sociale, Corbaccio, Milano 1925; Economia e corporativismo, Sate, Ferrara 1929; Intorno al principio formale della politica economica corporativa, Nistri-Lischi, Pisa 1930; Corporazioni, costi, prezzi e consumatori, Nuovi problemi, Ferrara 1935; Polemiche della «anumanità» della scienza economica e del corporativismo, Nuovi problemi, Ferrara 1935; Scienza economica pura, politica economica pura e corporativismo, Nuovi problemi, Ferrara 1937.

56 Cfr. Asgoi, Matricola.

57 Fra le sue opere La teoria del valore di C. Marx, Sandron, Milano-Palermo 1899; Sul principio regolatore della finanza pubblica, E. Croce, Napoli 1902; La speculazione economica, Società editrice libraria napoletana, Napoli 1907; Il capitalismo. Lineamenti storici, Bocca, Torino 1910; Economia, sindacalismo, socialismo, Società editrice partenopea, Napoli 1913; Manuale di economia politica, Morano, Napoli 1919; Il valore della scienza economica. Introduzione a una critica dell'economia politica, Morano, Napoli 1921; Finanza ed economia. Saggi, Morano, Napoli 1925; Al di là del capitalismo e del socialismo, Editrice Faro, Roma 1946.

58 Cfr. Asgoi, Matricola. Su di lui rinvio alla mia voce nel Dizionario biografico degli italiani, vol. LXII, Istituto della Enciclopedia italiana, Roma 2004.

59 Fra le sue opere Il sindacato del ferro in Italia, Roux e Viarengo, Torino 1899; Les conditions économiques, politiques et sociales de l'Italie pendant 1904-1905, Davy, Paris 1905. 
Fu iniziato nella Loggia Carlo Pisacane di Roma il 6 marzo $1908^{60}$.

9) Emanuele Sella (1879-1946), laureatosi nel 1902 in Giurisprudenza a Torino, fu professore di economia politica nelle Università di Perugia, Sassari, Cagliari, Parma, Messina e dal 1924 Genova, dove ricoprì la carica di rettore fra l'agosto e il settembre 1943, nel breve periodo racchiuso tra la caduta del fascismo e la nascita della Repubblica sociale italiana ${ }^{61}$.

Fu iniziato nella Loggia Francesco Guardabassi di Perugial'8 gennaio $1908^{62}$.

10) Giuseppe Testoni (1877-1957). Non propriamente un economista, ma inserito in questo elenco perché dopo la laurea in chimica all'Università di Bologna nel 1899 e il primo impiego come assistente nel locale Istituto di chimica generale, cominciò a occuparsi dei problemi della merceologia. Nel 1917 conseguì la libera docenza in chimica bromatologica e nel 1928 la cattedra di merceologia nell'Istituto superiore di scienze economiche e commerciali di Bari, da cui passò poi a Trieste e infine a Bologna, dove fondò l'Istituto di merceologia. Testoni fu nominato rettore dell'allora Istituto superiore di scienze economiche e commerciali e successivamente, quando tale Istituto divenne facoltà universitaria, ne fu preside per molti anni ${ }^{63}$.

Fu tra i membri della Loggia VIII Agosto di Bologna fondata nel $1886^{64}$.

11) Filippo Virgili (1865-?). Esponente di primo piano del movimento socialista senese, fu eletto consigliere comunale nel 1895, carica che mantenne ininterrottamente fino al 1914. Chiamato nel 1891 ad assumerel'incarico dell' insegnamento della statistica nell'Università di Siena, soltanto nel 1903, e dopo molte insistenze e vari concorsi andati a vuoto, ottenne finalmente la sospirata nomina a ordinario, proprio nel medesimo anno in cui fu affiliato col grado di maestro nella Loggia Arbia. Fu rettore dell'Università di Siena dal 1908 al $1910 \mathrm{e}$ quasi nello stesso periodo venerabile della sua loggia, carica che tornò a ricoprire nel 1919 e 1920. Nel 1905 fu protagonista di un episodio che ebbe risonanza anche a livello nazionale. Nel momento in cui il Partito socialista lanciò il pri-

Cfr. Asgoi, Matricola.

${ }_{61}$ Fra le sue opere L'uomo e la valanga. In difesa di Maffeo Pantaleoni, Tip. Amosso, Biella 1902; Studi sugli effetti della speculazione cerealicola, Bocca, Torino 1904; Speculazione commerciale e le crisi di produzione, Bocca, Torino 1906; La concorrenza. Sistema e critica dei sistemi, 2 voll., Bocca, Torino 1915-1916; Lezioni di scienza delle finanze, Assec, Genova 1925; Dottrina dei tre principii. Lezioni di economia politica, Cedam, Padova 1930; Dottrina del valore. Lezioni di economia ipostatica, Cedam, Padova 1931. Su di lui cfr. M. Vaudano (a cura di), La figura e l'opera di Emanuele Sella, Sella di Monteluce Foundation, Docbi-Centro studi biellesi, Biella 1999.

${ }^{62}$ Cfr. Asgoi, Matricola.

${ }^{63}$ Fra le sue opere Merceologia delle sostanze grasse e delle cere, La Grafolito, Bologna 19341935; Merceologia delle fibre tessili, La Grafolito, Bologna 1935; Corso di merceologia, Ed. universitarie, Bologna 1944.

64 Cfr. C. Manelli, La massoneria a Bologna dal XVIII al XX secolo, Edizioni Analisi, Bologna 1980, p. 139. 
mo referendum per stabilire l'incompatibilità dell'appartenenza alla massoneria per i propri iscritti, egli non esitò a dimettersi dal Psi. Nel 1913 aderì poi al Partito socialista riformista e fu tra i principali fautori della lista bloccarda che si costituì a Siena in vista delle elezioni amministrative del $1914 \mathrm{e}$ che risultò nettamente sconfitta dalla coalizione clerico-moderata ${ }^{65}$.

Come si vede da questo elenco, anche fra i docenti universitari di materie economiche o statistiche la massoneria seppe reclutare figure di notevole rilievo sia per il profilo scientifico, sia per i ruoli occupati nei vari atenei, sia per gli incarichi politici o istituzionali ricoperti da molti di essi. Si tratta di un nucleo relativamente esiguo, che tuttavia, attraverso indagini più approfondite condotte sugli elenchi dei professori universitari e su quelli degli iscritti alle varie obbedienze massoniche, potrebbe essere ulteriormente incrementato. In ogni caso già questa lista di nomi, per quanto parziale, rivela una capacità attrattiva della massoneria su esponenti di primo piano della cultura economica italiana fra le due guerre che pare degna di nota.

\section{Considerazioni conclusive}

Che cosa possiamo dunque dire, in conclusione, circa il ruolo esercitato dalla massoneria nell'università italiana della prima metà del Novecento? Quale significato attribuire, in particolare, a questa qualificata presenza di liberi muratori (o di ex liberi muratori) nel mondo giuridico ed economico dell'Italia fascista? Le opinioni anche fra gli osservatori contemporanei erano affatto divergenti. Secondo il pedagogista Giuseppe Lombardo Radice, i massoni si muovevano come una falange compatta, come una società di mutuo soccorso insediata dentro l'apparato statale che controllava le più delicate dinamiche della vita accademica, a cominciare dai concorsi e dalle chiamate in cattedra. In più articoli pubblicati sulla rivista «Nuovi doveri», da lui fondata nel 1907, Lombardo Radice denunciò l'azione nefasta svolta dalla massoneria, sebbene soprattutto con riferimento al mondo della scuola che egli meglio conosceva:

Massoni sono stati i peggiori ministri dell'Istruzione Pubblica, massoni sono quasi tutti i non mai abbastanza lodati grafficarte della burocrazia Minervina; di massoni è formata la gran maggioranza dei capi d'Istituto anziani e semi anziani, la cui opera è stata finora disastrosa per la scuola; [...] massoni quegli insegnanti che si misero a capo - a suo tempo - della battaglia contro la legge sullo stato giuridico che cercava di assicurare l'indipendenza dall'arbitrio ministeriale e l'onesto reclutamento degli insegnanti; massone era il Ministro della Pubblica

65 Su di lui cfr. G. De Plato, Per una biografia di Filippo Virgili, professore di statistica, «Le carte e la storia», 5 (2), 1999, pp. 155-166. Sulla sua militanza massonica cfr. F. Conti, Logge e massoni a Siena e nel Senese dall'Unità alla Grande guerra, in V. Serino (a cura di), I maestri del tempio. Logge e Liberi Muratori a Siena dall'Illuminismo all'avvento della Repubblica, il Leccio, Monteriggioni 2003, pp. 77-98. 
Istruzione che gridava tutti i momenti di avere 'le mani legate' e di non potere perciò abilitare, nominare, traslocare gli insegnanti, e riempire di fratelli i musei e le pinacoteche del bel regno ${ }^{66}$.

Lombardo Radice combinava in quegli anni una visione pedagogica di tipo idealistico, molto vicina a quella di Giovanni Gentile, con una tensione eticopolitica di matrice salveminiana che lo portò ad aderire al Partito socialista e a nutrire nei confronti della massoneria una profonda avversione. Diverso era il giudizio di Arturo Carlo Jemolo, anche lui peraltro non certo sospettabile di atteggiamenti indulgenti verso l'istituzione liberomuratoria, che in un libro di memorie ebbe a scrivere:

Anche fuori del ristretto campo della burocrazia ho l'impressione che la massoneria non potesse più di quel che può ogni partito che partecipa al potere: spingere avanti qualcuno dei suoi, ma con una spinta molto moderata. [...] È possibile fosse la massoneria a riuscire a creare una certa risonanza, una certa popolarità a qualcuno di quei palloni gonfiati (un professore della Facoltà di lettere di Roma degli anni di Umberto I); ma nella università, nei ministeri, dovunque, salivano anche in alte posizioni cattolici a viso aperto, conservatori, uomini alienissimi dalla mentalità massonica. Sfogliare annuari mostra proprio quanto sia ingiusta la deformazione per cui in certi periodi la massoneria non avrebbe permesso agli avversari di alzare il capo. [...] Inoltre per questa limitazione ad agire, direttamente solo sui suoi, non poteva esplicare l'azione capillare che oggi compiono i partiti o certe grandi associazioni, con un comune denominatore ben più largo. Penso ad es. a quello che oggi possono nel mondo universitario i professori cattolici, con le indicazioni che danno per ogni elezione del Consiglio Superiore, per ogni votazione di commissioni. La massoneria non poté mai concepire campi di azione così vasti. Gli ambiti dove più si affermava - le università popolari, gli enti e le compagnie assicurative, le facoltà di medicina - non erano centri di potere ${ }^{67}$.

Ulteriori e più analitiche ricerche condotte negli archivi delle università e sulle carte dei singoli docenti potranno darci risposte più fondate ed esaurienti di quelle che siamo oggi in grado di offrire. L'impressione però, almeno per il periodo di cui qui ci siamo occupati, è che avesse più ragione Jemolo di Lombardo Radice. È assai probabile che in alcuni contesti locali dove la massoneria era più radicata vi siano state carriere ed elezioni a cariche direttive che beneficiarono di accordi maturati nel segreto delle riunioni di loggia. Tuttavia, i vertici del Goi e poi anche quelli della Gran Loggia d'Italia di Piazza del Gesù non dettero mai l'idea di avere una precisa politica universitaria né rivelarono la volontà di utilizzare gli acca-

66 G. Lombardo Radice, La massoneria, in Id., Saggi di propaganda. Politica e pedagogica (19071910), Sandron, Milano 1910, p. 24. Si veda inoltre Id., Clericali e massoni di fronte al problema della scuola, La Voce, Roma 1920.

67 A.C. Jemolo, Anni di prova, Passigli, Firenze 1991 (prima ed. Neri Pozza, Venezia 1969), pp. 115-117. 
demici e gli intellettuali come soggetti organici alle obbedienze per realizzare gli obbiettivi che esse si prefiggevano. Agli universitari la massoneria chiese soprattutto di condividere i suoi obbiettivi sociali e culturali, e di sostenerli mediante la partecipazione a varie associazioni 'profane' che erano emanazione delle logge: società di pubblica assistenza, società per la cremazione, comitati per la pace $\mathrm{e}$ per l'abolizione della pena di morte, scuole e università popolari, banche del popolo, comitati per il matrimonio civile e per il divorzio, circoli laici e anticlericali di varia natura. Attraverso questo reticolo associativo la massoneria fu in grado di moltiplicare la sua influenza sulla società civile e sulla politica, e indubbiamente in tali contesti il fatto di poter contare sul sostegno di autorevoli esponenti della comunità scientifica ebbe un peso considerevole. Oltre a questo coinvolgimento strutturale e duraturo sono da segnalare soltanto iniziative estemporanee, come quella che prese corpo nel dicembre 1923, mentre la Chiesa stava definendo i suoi programmi per il giubileo del 1925 e il Goi pensò di «contrapporgli un Congresso di scienziati convocati da tutto il mondo». In tale circostanza il gran maestro Domizio Torrigiani propose di istituire una «commissione di scienziati italiani» che assumesse l'iniziativa e furono fatti i nomi «degli illustri professori Bianchi, Lustig, Paternò, Sraffa $\gg^{68}$, tutti massoni o ex massoni ritenuti evidentemente assai vicini a Palazzo Giustiniani. Il progetto, però, cadde nel vuoto.

Niente a che vedere insomma, per quanto riguarda l'età liberale, rispetto alle ben più ambiziose forme di mobilitazione che il Grande Oriente d'Italia avrebbe avviato nel secondo dopoguerra, quando nel maggio $1954 \mathrm{fu}$ addirittura organizzato un 'primo convegno nazionale massonico dei professori e docenti universitari'. Svoltosi a Roma, vide la partecipazione di un numero imprecisato di professori, i quali, a partire da due relazioni presentate da Giunio Bruto Crippa e da Ugo Della Seta, si confrontarono sui vari problemi dell'università e sul sostegno da dare a quelle strutture associative, in primis la Corda Fratres, che erano diretta emanazione della massoneria ${ }^{69}$.

Infine, un'ultima considerazione che rinvia alle ragioni e alle finalità specifiche di questo contributo. Esso nasce con un intento che potremmo definire 'ancillare' rispetto alle ricerche degli studiosi di storia del pensiero giuridico ed economico, o di quelli che si occupano di storia delle università e delle istituzioni. Non si è posto ovviamente l'obbiettivo di ricostruire il profilo intellettuale e scientifico dei giuristi o degli economisti menzionati nelle pagine precedenti, né chi scrive

68 Asgoi, Verbali della giunta, 13 dicembre 1923. Su questa fase della storia della massoneria italiana e per ulteriori riferimenti bibliografici cfr. F. Conti, Massoneria e fascismo: dalla marcia su Roma alla legge sulle associazioni segrete, in Id. (a cura di), La massoneria italiana da Giolitti a Mussolini. Il gran maestro Domizio Torrigiani, Viella, Roma 2014, pp. 85-108.

69 Cfr. Primo convegno nazionale massonico dei professori e docenti universitari. Maggio 1954. Relazioni di Giunio Bruto Crippa e Ugo Della Seta, Tip. Bardi, Roma 1954. Sulla Corda Fratres cfr. A.A. Mola, Corda Fratres. Storia di una associazione internazionale studentesca nell'età dei grandi conflitti, 1898-1948, Clueb, Bologna 1999. Sui problemi della docenza universitaria nell'immediato secondo dopoguerra cfr. G. Montroni, La continuità necessaria. Università e professori dal fascismo alla Repubblica, Le Monnier, Firenze 2016. 
avrebbe avuto le competenze necessarie per farlo. Si è limitato a segnalare, sulla base di precisi riscontri documentari, la loro appartenenza alla massoneria, muovendo dalla convinzione che in quegli anni questo dato avesse un significato rilevante, fosse rivelatore di scelte personali e orientamenti valoriali ben precisi.

Credo infatti che sia difficile derubricare la decisione di aderire alla massoneria da parte di questi illustri esponenti della cultura italiana della prima metà del Novecento come scelta meramente opportunistica oppure come coinvolgimento inconsapevole e accidentale nelle attività di un sodalizio, di cui non si conoscevano scopi e fini. Logiche di carriera e ripercussioni sull'adesione alla massoneria dell'appartenenza a cordate accademiche probabilmente vi furono. E potranno essere appurate soltanto da indagini più approfondite che ricostruiscano la trama delle relazioni e delle corrispondenze epistolari fra i diversi individui. L'impressione però, come rivelano altri passaggi del percorso esistenziale dei tanti docenti universitari qui citati, è che l'affiliazione massonica fu per essi quasi sempre una scelta ben ponderata, fondata su solidi presupposti ideali e politici. Per tutti loro rappresentò la manifestazione di una ben definita volontà di riconoscersi nei valori della laicità, nel mito positivistico del progresso, nell'idea che la scienza avrebbe trionfato contro tutte le forze dell'oscurantismo, in un patriottismo di sapore risorgimentale e romantico, in un solidarismo umanitario volto a garantire la graduale emancipazione delle classi popolari senza sussulti rivoluzionari che mettessero a rischio gli equilibri sociali e politici vigenti. Questo fu l'universo ideale della massoneria nel periodo che va dall'Unità al fascismo e in esso decine e decine di professori universitari, spesso autentici luminari dell'accademia italiana, trovarono un motivo di attrazione, un riferimento identitario, un modo per affermare una scelta di campo ben precisa: a favore del democraticismo progressista, laico e anticlericale, e contro ogni conservatorismo, a cominciare da quello della Chiesa cattolica e delle forze di orientamento nazionalista e reazionario.

Un ultimo dato da prendere in considerazione riguarda il fatto che quella di docente universitario era all'epoca una professione realmente itinerante. La carriera universitaria comportava lunghi periodi di ricerca e d'insegnamento trascorsi in sedi diverse, con frequenti spostamenti in Italia e all'estero. Ebbene, la massoneria era in grado di offrire un network di relazioni sovranazionali, una rete di accoglienza che poteva facilitare l'inserimento nelle varie città in cui si era costretti a trasferirsi, immediati contatti con quei colleghi che condividevano l'affiliazione e che sovente, come si è visto, ricoprivano incarichi direttivi sia negli atenei che nelle singole facoltà. E quindi il solidarismo massonico poteva garantire aiuti, agevolazioni, protezione. Anche questo dovette essere un fattore non del tutto irrilevante nello spingere alcuni docenti alla scelta di affiliarsi alla massoneria. Del resto, qualcosa di analogo accadde nello stesso periodo per altre categorie socio-professionali, come i militari e gli artisti di teatro, che erano anch'esse caratterizzate da una forte mobilità territoriale e il cui peso negli elenchi degli iscritti al Grande Oriente d'Italia appare decisamente sovradimensionato ${ }^{70}$. 